\title{
Applying Behavioral Economics and Neuroeconomics to Medical Education and Clinical Care
}

\author{
Gustavo Saposnik
}

Keywords: Neuroeconomics, Postgrad medical education, Multiple sclerosis, Stroke, Decision-making, Therapeutics

doi:10.1017/cjn.2018.371

Can J Neurol Sci. 2019; 46: 35-37

Education is the most powerful weapon which you can use to change the world.

Nelson Mandela (1918-2013)

\section{INTRODUCTION}

Behavioral economics (BE) studies the effects of psychological, cognitive, emotional, and social factors on the economic decisions of individuals and institutions. Neuroeconomics-also called decision neuroscience-is the science that studies the principles of how the brain underpins economic and other decisions. ${ }^{1}$ In the last few years, some researchers have applied concepts from BE and neuroeconomics to clinical care and gained novel insights. Several studies showed the impact of cognitive biases (e.g. overconfidence, status quo, outcome bias, etc.) and risk preferences (e.g. aversion to ambiguity, tolerance to uncertainty, etc.) in clinical care. ${ }^{2}$ This is likely related to physicians' limited education in decision-making and formal training in risk management. Most medical schools do not include decisionmaking and risk management courses in their curriculums. ${ }^{3}$ Medical education in these two crucial topics (commonly faced by physicians in their routine clinical practice) is key for the training of future physicians who would be making rationale and complex decisions about others (e.g. their patients).

\section{DECISION-MAKING UNDER UNCERTAINTY}

Most physicians must make diagnostic and therapeutic decisions under uncertainty with insufficient or imperfect information. As such, individual physicians' tolerance to uncertainty may affect decisions in clinical care. In BE, uncertainty is a generic term that comprises risk and ambiguity. Risk applies to events with known probability, whereas ambiguity is a term reserved for events for which probabilities are unknown. ${ }^{1}$ For example, the prevention of stroke with anticoagulants for patients with atrial fibrillation is a classic example of risk, given the probability is known (approximately 5\%/year without taking into account specific characteristics). Some therapeutic decisions (e.g. treatment response for MS) would fall under ambiguity, given that the true efficacy of an agent is not known at the individual patient level.
Typically, people are reluctant to take risks or make choices under ambiguity.

Ambiguity is not an all or nothing concept and it can be studied using experimental designs. For example, previous studies included experiments asking participants to choose between a visual option with known 50/50 probability of winning $\$ 400$ or $\$ 0$ versus an option with unknown probability of the same outcomes. Grey bars represented the degree of ambiguity to which the winning probability was unknown (Figure 1). ${ }^{5}$ The degree of ambiguity aversion can be defined as the proportion of times participants chose the 50/50 option over the ambiguous option involving the same outcomes.

A similar strategy can be used for assessing ambiguity in the health domain. For example, participants can be asked to choose between Treatment A (50\% probability of survival) and Treatment B (the probability of survival is unknown), with the grey bars quantifying how much is unknown about the probability of survival. Another factor that may influence clinical decisions is risk aversion defined, for example, as the tendency to prefer safe payoffs over probabilistic payoffs when the expected value of both options is identical. $^{4}$

A risk-averse patient would thus prefer a treatment that provides a small improvement with certainty over a treatment that provides a larger or no improvement with equal chance (50/50). For example, participants may choose to receive a secured $\$ 100$ instead of the 50/50 chance of winning $\$ 400$ or $0 \$$ (with a higher expected value of \$200).

\section{Application to Medical Decision-making}

Although most studies in medical decision-making have targeted patients, few reports were focused on physicians. Moreover, there are a few studies applying experiments from BE or neuroeconomics to medical care. For example, a study including 94 obstetricians who attended 3488 deliveries evaluated the tolerance

From the Department of Economics, University of Zurich; Outcomes Research and Decision-Neuroscience Unit, Department of Medicine, St Michael's Hospital, University of Toronto, Toronto, Canada; Institute for Clinical Evaluative Sciences (ICES), Toronto, Canada.

Received September 10, 2018. Final Revisions Submitted October 1, 2018. Date of ACCEPTANCE OCtOBER 15, 2018.

Correspondence to: G. Saposnik, University of Toronto, PhD Candidate in

Neuroeconomics, University of Zurich. Email: saposnikg@smh.ca 


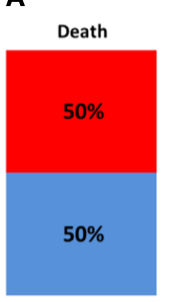

Survival

Treatment A

(exact probability $\quad$ (unknown probability of survival represented by the grey bars) of survival)

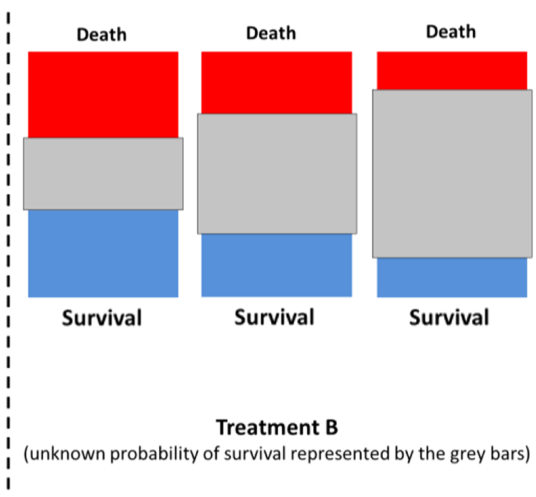

B

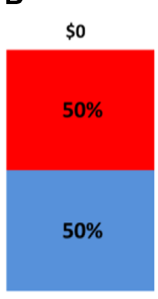

$\$ 400$

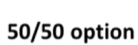

(exact probability)

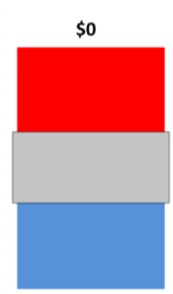

$\$ 400$

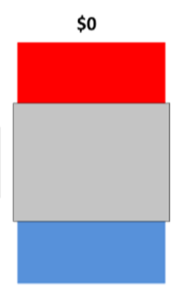

$\$ 400$

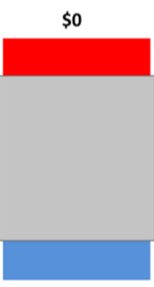

$\$ 400$

Figure 1: Experiments to determine aversion to ambiguity. ${ }^{5}$

Panel A, Varying ambiguity in the health domain. Panel B, Varying ambiguity in the financial domain.

to uncertainty using validated survey. The authors showed that stronger tolerance of ambiguity was associated $(p<.006)$ with optimal management (reflected by lower instrumental vaginal deliveries) and lower medical errors. ${ }^{6}$ Another study randomized primary care clinicians to one of three interventions (an computerized alert system, a peer comparison, control group) regarding antibiotic prescriptions for patients with an acute respiratory infection. Antibiotics are deemed not necessary (and considered inappropriate use) for most upper respiratory tract infections. The peer comparison group was informed about their performance compared to their colleagues (e.g. 'Top 10 of their peers' when making correct decisions not prescribing antibiotics), whereas the computerized alert group received alternative options. ${ }^{7}$ Given the immediate feedback given to all participants, the authors intrinsically applied the concepts of peer pressure and herding (participants followed recommendations from other colleagues rather than deciding independently on the basis of their own private information) to the peer comparison group. Primary care physicians randomized to the peer comparison group had a reduced prescription of antibiotics (odds ratio [OR] 0.73; 95\% confidence interval [CI] 0.53-0.995). ${ }^{7}$

Our group studied therapeutic decisions in multiple sclerosis (MS) made by neurologists applying experiments evaluating risk, ambiguity, and herding. ${ }^{5}$ There are many treatments available for MS, ranging from low efficacy and low risk of side effects to highly efficacious agents with greater risk of severe side effects. We found that 7 out of 10 neurologists from Spain $(n=96)$ failed to escalate treatment when recommended by guidelines, a phenomenon called therapeutic inertia (TI). In other words, physicians with TI elected the status quo by not changing the treatment when patients were not doing well. Aversion to ambiguity in the financial domain was the most relevant predictor of TI (OR 7.39; 95\% CI 1.40-38.9), followed by low tolerance to uncertainty (OR 3.47 ; $95 \%$ CI $1.18-10.2) .^{5}$ This finding was replicated among neurologists from Argentina and Chile $(n=115$; OR for aversion to ambiguity: $2.91 ; 95 \%$ CI $1.06-8.04$; OR for low tolerance to uncertainty: 1.47 ; 95\% CI 1.02-2.11). Similar associations were found for stroke prevention in the management of atrial fibrillation (most common cardiac arrhythmia) among cardiologists and general practitioners.

In a follow-up study, the authors evaluated the potential benefits of an educational intervention to ameliorate TI. We applied the traffic light system (TLS) as an educational strategy to facilitate the decision-making process of treatment selection for MS by reducing aversion to ambiguity. The TLS emerged as a strategy to warn people and draw their attention to risk, with the goal of reducing human errors by facilitating the integration of specific situations with a corresponding action. The expectation is that the TLS would decrease aversion to ambiguity and consequently lower TI. The authors evaluated the association between risk preferences and aversion to ambiguity with physicians' responses to different simulated situations of MS patients at low, medium, or high risk of disease progression. ${ }^{5}$ The goal of the TLS was to match case scenarios with three types of situations according to the risk of disease progression: red light (high risk of progression, "stop and think"), yellow light (intermediate risk of progression, "reassessment needed sooner than usual"), and green light (low risk of progression, "continue the same strategy"). The application of the TLS was feasible and promising by showing a trend towards a reduction in TI compared to controls (OR $0.57 ; 95 \%$ CI $0.26-1.22) .{ }^{8}$ In another study, the authors assessed herding among 96 neurologists with expertise in MS. Herding is a phenomenon that can occur in MS when neurologists follow a therapeutic recommendation by a colleague even though it is not supported by best practice clinical guidelines. Nearly 8 out of 10 neurologists exhibited herding. Higher volume of medical consultations per week was associated with higher incident risk of herding (OR $1.08,95 \%$ CI 1.01-1.14). Demographic factors, years of practice, and physicians risk preferences were not associated with herding. These findings may suggest that physicians when overwhelmed by the volume and complexity of medical consultations are more likely to follow peers' recommendations (even when erroneous) instead of individually analyzing medical information. The most common medical environment that may lead to herding-like decisions are emergency departments prone to cascade of errors due to high volume of consultations, time pressures, physicians' frequent shifts leading to fragmented care and suboptimal communication.

Together, these examples provide some evidence for the link between physicians' aversion to ambiguity, herding, and suboptimal treatment decisions, ultimately affecting patient outcomes. Furthermore, physicians exposed to cognitive biases or TI could be trained by implementing effective educational interventions. 


\section{What are the Underlying Biological Basis That Link Ambiguity Aversion, the TLS, and TI?}

A study applying neuroeconomic methods (functional MRI) in food choice showed that the TLS enhances the coupling between brain regions associated with valuation and self-control. Specifically, the red traffic light activated the left inferior frontal gyrus and the dorsolateral prefrontal cortex, a brain region implicated in self-control of food choice. In other words, the red traffic light may interrupt automatic decisions (e.g. temptation to choose tastier but unhealthier food, the status quo of no treatment changes) by triggering a re-evaluation process (e.g. rethinking food choices under the aspect of health being valued more strongly than taste, rethinking about the risk of disease progression), allowing for deliberation to take place. ${ }^{9}$

\section{Closing Remarks}

Both $\mathrm{BE}$ and neuroeconomics have provided useful insights into medicine. Physicians are the most responsible decisionmakers, sometimes with limited training in risk management and medical education. Future studies evaluating educational interventions may help improve physicians' and patients' treatment choices, leading to better outcomes and quality of life.

\section{ACKNOWLEDGEMENTS}

The author thanks Prof. Philippe Tobler for his critical comments and suggestions regarding the content of this article.

\section{Disclosures}

Dr. Saposnik is supported by the Heart and Stroke Foundation of Canada (HSFC) Career Scientist Award. He has received operating grants from Roche, Boehringer Ingelheim, HSFC, Ontario Stroke Network.

\section{REFERENCES}

1. Platt ML, Huettel SA. Risky business: the neuroeconomics of decision making under uncertainty. Nat Neurosci. 2008;11 (4):398-403.

2. Saposnik G, Redelmeier D, Ruff CC, Tobler PN. Cognitive biases associated with medical decisions: a systematic review. BMC Med Inform Decis Mak. 2016;16(1):138.

3. Dijkstra IS, Pols J, Remmelts P, Brand PL. Preparedness for practice: a systematic cross-specialty evaluation of the alignment between postgraduate medical education and independent practice. Med Teach. 2015;37(2):153-61.

4. Camerer C, Weber M. Recent developments in modeling preferences: Uncertainty and ambiguity. J Risk Uncertain. 1992;5(4): 325-70.

5. Saposnik G, Sempere AP, Prefasi D, et al. Decision-making in Multiple Sclerosis: The Role of Aversion to Ambiguity for Therapeutic Inertia among Neurologists (DIScUTIR MS). Front Neurol. 2017;8:65.

6. Yee LM, Liu LY, Grobman WA. The relationship between obstetricians' cognitive and affective traits and their patients' delivery outcomes. Am J Obstet Gynecol. 2014;211(6):692 e691-6.

7. Persell SD, Doctor JN, Friedberg MW, et al. Behavioral interventions to reduce inappropriate antibiotic prescribing: a randomized pilot trial. BMC Infect Dis. 2016;16:373.

8. Saposnik G, Maurino J, Sempere AP, et al. Overcoming Therapeutic Inertia in Multiple Sclerosis Care: A Pilot Randomized Trial Applying the Traffic Light System in Medical Education. Front Neurol. 2017;8:430.

9. Enax L, Krajbich I, Weber B. Salient nutrition labels increase the integration of health attributes in food decision-making. Judgm Decis Mak. 2016;11(5):460-71. 